\title{
Patient Evaluation of Satisfaction and Outcomes with an Autoinjector for Self-Administration of Subcutaneous Belimumab in Patients with Systemic Lupus Erythematosus
}

\author{
Ebony Dashiell-Aje ${ }^{1,5} \cdot$ Gale Harding1 $^{1}$ Katie Pascoe ${ }^{2} \cdot$ Jane DeVries $^{2} \cdot$ \\ Pamela Berry $^{3} \cdot$ Sulabha Ramachandran ${ }^{4}$
}

Published online: 27 September 2017

(C) The Author(s) 2017. This article is an open access publication

\begin{abstract}
Objective This study assessed patient experiences of using an autoinjector device to self-administer subcutaneous belimumab for the treatment of systemic lupus erythematosus (SLE). Satisfaction, ease and convenience of use, and confidence with the device were assessed, in addition to overall experience with belimumab.

Methods This cross-sectional study was conducted among patients who completed a phase IIb open-label, multi-dose usability, tolerability, and safety study of subcutaneous belimumab (NCT02124798), in which patients receiving intravenous belimumab or subcutaneous belimumab using a prefilled syringe were switched to eight weekly self-administered doses of subcutaneous belimumab using the autoinjector. This follow-up study comprised an online/paper questionnaire and qualitative telephone interviews.

Results In total, 43 patients receiving belimumab completed the questionnaire, 21 of whom also completed a follow-up telephone interview. Qualitative interviews indicated that 17 of 21 (81\%) patients had a positive experience using the autoinjector; all patients considered the autoinjector to be convenient. Of the 42 patients who
\end{abstract}

Electronic supplementary material The online version of this article (doi:10.1007/s40271-017-0276-2) contains supplementary material, which is available to authorized users.

\footnotetext{
Ebony Dashiell-Aje

Ebony.Dashiell-Aje@fda.hhs.gov

1 Evidera, Bethesda, MD, USA

2 Value Evidence and Outcomes, GlaxoSmithKline, London, UK

3 GlaxoSmithKline, Collegeville, PA, USA

4 Value Evidence and Outcomes, GlaxoSmithKline, King of Prussia, PA, USA

510903 New Hampshire Ave, Silver Spring, MD 20993, USA
}

switched from intravenous belimumab to the autoinjector, $32(76 \%)$ expressed a preference for the autoinjector over intravenous administration; reasons included convenience, time saved, cost, and reduced injection pain. The most commonly reported disadvantage of the autoinjector was injection discomfort ( $n=5$ [24\%]; qualitative interview). Compared with intravenous administration, the autoinjector improved ability to work (17 of 29 [59\%] of those employed) and carry out daily activities (40\%). Conclusion Patients with SLE reported high levels of satisfaction with the belimumab autoinjector and preferred the autoinjector to intravenous administration, citing advantages such as time saved, cost, and improved ability to work and carry out daily activities.

\section{Key Points for Decision Makers}

Patient satisfaction with new treatments is critical, and effective convenient treatments that integrate well into patients' lives contribute toward this. Overall patient satisfaction was favorable for the belimumab autoinjector compared with intravenous administration of belimumab.

Patients considered the belimumab autoinjector to be more convenient than intravenous belimumab because of the shorter administration time, decreased travel time, portability, and reduced/no pain.

Regardless of administration route, many patients reported improvements in activities of daily living with belimumab. Furthermore, compared with intravenous administration, 59\% of employed patients stated that the autoinjector improved their ability to work and $40 \%$ reported that their ability to carry out daily activities improved. 


\section{Introduction}

Intravenous administration of belimumab $10 \mathrm{mg} / \mathrm{kg}$ plus standard of care (SoC) for systemic lupus erythematosus (SLE) is licensed for use in over 60 countries (including countries in Europe, the USA, Canada, and Australia) for the treatment of SLE in adults with active, autoantibodypositive disease [1]. Administration of intravenous belimumab requires patients to visit a clinic or infusion center every 4 weeks. A study evaluating satisfaction with belimumab and SLE treatment indicated that $>50 \%$ of patients receiving intravenous belimumab would prefer to self-administer their treatment at home; this preference was more common among patients who were employed or were students [2]. Self-administration could potentially save time and reduce costs for both patients and the health service [3]. However, currently belimumab is only available by intravenous administration in a clinic.

An autoinjector device and prefilled syringe have been developed for subcutaneous administration of belimumab to enable self-administration of treatment. Initial trials of a single dose of subcutaneous belimumab in healthy volunteers, self-administered using the autoinjector or prefilled syringe, demonstrated acceptable pharmacokinetic, tolerability, reliability, usability, and safety profiles [4]. A subsequent phase II, open-label, single-arm, multi-dose study of subcutaneous belimumab in patients with SLE in real-life conditions (GSK study 200339; NCT02124798) demonstrated that the autoinjector was reliable and well tolerated for home administration [5]. Patient assessments indicated a good level of usability and safety was consistent with the known safety profile of belimumab. Reported pain on injection was low and decreased with repeated administration as patients gained experience with using the device.

The efficacy and safety of subcutaneous belimumab using a prefilled syringe plus $\mathrm{SoC}$ was demonstrated in a 52-week, phase III, randomized, double-blind, placebocontrolled pivotal trial (BLISS-SC) [6]. Results from this study were consistent with outcomes observed in the phase III BLISS studies among adults treated with intravenous belimumab plus SoC $[7,8]$.

Compared with other routes of administration, subcutaneous treatment formulations can have advantages in terms of convenience, ease of use, and the possibility of self-administration [3]. However, there may also be disadvantages, including anxiety and adverse injection-site reactions [3]. Evidence comparing patients' perspective of subcutaneous and intravenous routes of administration is lacking [3]. Herein, we discuss the results from a follow-up of the phase II study that assessed patient experience and satisfaction with the autoinjector and the impact of adding subcutaneous belimumab to SoC.

\section{Patients and Methods}

\subsection{Objectives}

The primary objectives of this study were to assess patients' experiences using the autoinjector to deliver belimumab, with regard to satisfaction, ease and convenience of use, confidence using the device, and their broader experience with belimumab. Secondary objectives were to explore patient experiences associated with switching to the autoinjector from intravenous or subcutaneous prefilled syringe administration, and patient preferences for administration routes. Exploratory subgroup analyses included investigating changes in symptoms related to duration of belimumab treatment, ability to work, and preference for route of administration.

\subsection{Study Design and Study Population}

This cross-sectional study recruited patients who completed the phase II multi-dose usability, tolerability, and safety study of subcutaneous belimumab via autoinjector (GSK Study 200339; NCT02124798) [5]. The design and inclusion/exclusion criteria of the parent study have been reported previously [5]: patients receiving intravenous belimumab $(n=93)$ or subcutaneous belimumab using a prefilled syringe $(n=2)$ were eligible to switch to subcutaneous belimumab using an autoinjector device. Following training on use of the autoinjector, patients selfadministered weekly doses of belimumab for 8 weeks, 4 with supervision at the clinic (at weeks 1,2, 4, and 8) and 4 at home (at weeks 3, 5, 6, and 7).

Patients were recruited to this follow-up study by site personnel at their week 8 (NCT02124798) visit or by telephone/in person following this visit.

The present study comprised two parts: a self-administered questionnaire followed by qualitative telephone interviews among a subset of patients who agreed to be contacted. Patients completed the questionnaire online (a paper-based version was provided if requested) within 14 days of their week 8 (NCT02124798) visit. Only data obtained within this time period were included in the primary analyses.

Patients who completed the questionnaire and indicated interest in a follow-up telephone interview were invited to participate in a pre-scheduled one-on-one telephone interview within 21 days of their week 8 (NCT02124798) visit; a sample of 30 patients was the target number of interviews. Patients were remunerated for completing the survey and interview.

Institutional review board approval was obtained at all sites prior to study initiation. Patients provided consent 
(online) to participate in the online questionnaire and written informed consent prior to paper questionnaire administration and telephone interviews.

\subsection{Questionnaire and Interview Development and Content}

The questionnaire and a semi-structured telephone interview guide were developed for this study based on a review of the literature and evaluation of SLE-specific patientreported outcome instruments [2, 9, 10]. The questionnaire comprised 43 items, including background information (two items), sociodemographic/clinical characteristics (two items), and the assessment of patients' experience with belimumab (11 items), experience with the autoinjector (nine items; in the Electronic Supplementary Material [ESM] 1), experience with intravenous belimumab (seven items), experience with belimumab administered by prefilled syringe (eight items), and impact of delivery method on health-related quality of life (HRQoL; i.e. work/daily activity performance [four items]). The majority of questions utilized Likert-scale responses, with patients required to recall experiences with belimumab since the beginning of treatment. The questionnaire responses were used to evaluate changes in symptoms, including the frequency and severity of flares, while using belimumab.

The semi-structured telephone interview guide comprised 24 open-ended questions, with instructions and probes to help the interviewer direct the discussion based on participant responses. This interview guide was designed to gain more detailed information about patients' experience with belimumab, including changes in symptoms (i.e., the frequency and severity of flares), the impact of belimumab treatment on HRQoL, patients' experience with switching administration routes, and preference for administration routes. The following is an example of an open-ended question included in the interview guide (further examples are provided in ESM 2):

Have you experienced any changes in your ability to engage in day-to-day activities since starting Benlysta [belimumab]? (use participant's responses to Q8 and Q9 of the questionnaire to help guide the discussion). If so, what changes have you experienced? Probe (if not mentioned): improvement or worsening in the following areas of life: work (e.g., attendance, performance); personal relationships (e.g., family, friendships); leisure (engaging in activities you enjoy); daily living (e.g., grooming, bathing, running errands).

Questionnaire responses were reviewed prior to the telephone interviews to inform the interviewer and to limit the duplication of questions. Interviews were designed to be completed within approximately $45 \mathrm{~min}$.

\subsection{Data Collection and Analyses}

Questionnaire data were collected via a web-based platform (with paper questionnaires provided upon request) through YouGov.com, a third-party research and consulting services agency, and exported to a central database for analysis. Paper questionnaire responses were double-entered and merged into the database with online responses. Demographic and clinical characteristics collected at baseline in study NCT02124798 were used to characterize the sample. Data from the questionnaire were analyzed using descriptive statistics ( $n$, mean, standard deviation [SD], and/or frequency). Planned exploratory subgroup analyses included duration of belimumab treatment, change in general symptoms, flare frequency, flare severity, and fatigue. Post hoc exploratory subgroup analyses included improvement in ability to work and preferred mode of administration.

Audio recordings of the telephone interviews were transcribed and patient identifiable information was removed prior to analysis. Personnel experienced in qualitative analysis methods analyzed the data using a studyspecific coding dictionary and content analysis approach. ATLAS.ti 7.1.8. software (Scientific Software Development $\mathrm{GmbH}$, Berlin, Germany) was used to create and organize concepts identified during the interviews to evaluate the underlying structure of the qualitative data. Table 1 in the ESM shows the reported frequency of the codes that support the data presented in this manuscript and provides sample quotes to support the most highly endorsed codes.

\section{Results}

\subsection{Patient Disposition}

A subset of the parent study sites was invited to join this study. In total, 95 patients participated in the parent study and 44 were invited to join this study. With one exception, all eligible patients agreed to take part in the questionnaire ( $n=43$; Fig. 1); however, one patient completed the survey outside of the required timeframe so was excluded from the primary analysis. The majority $(n=41)$ of patients switched directly from intravenous belimumab to the autoinjector; of these, one had previous experience of the subcutaneous belimumab prefilled syringe. Two patients switched directly from subcutaneous belimumab prefilled syringe to the autoinjector. 


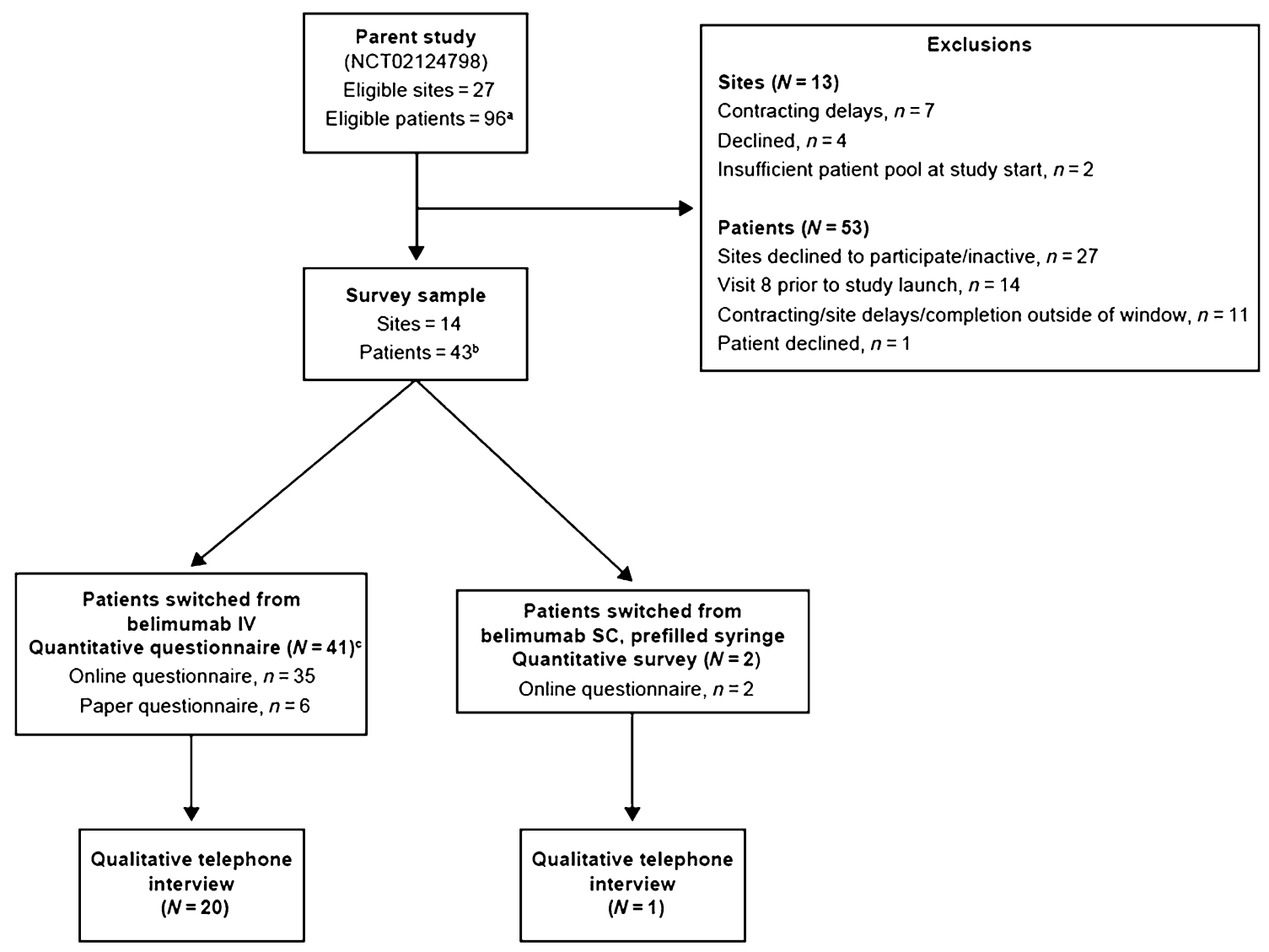

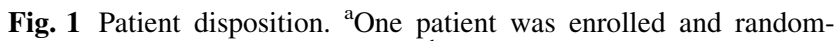
ized, but did not initiate treatment; ${ }^{b}$ one patient completed the survey outside of the required time frame so was excluded from the primary

Patient characteristics were representative of those who completed study NCT02124798 (Table 1) [5]. All patients who participated in the survey completed the eight weekly autoinjector doses (Fig. 1). The majority of patients $(n=30[71 \%])$ had received belimumab for $>1$ year.

\subsection{Patient Experience with Using the Autoinjector}

According to the questionnaire data, all patients were satisfied $(n=5[12 \%])$ or very satisfied $(n=37[88 \%])$ with the training they received before using the autoinjector. Patients felt confident in their ability to use the autoinjector correctly the first time they used it on their own outside of the clinic (Fig. 2). At the end of the study, 35 (83\%) patients were extremely confident they could use the autoinjector correctly. During qualitative interviews, patients stated that their confidence was due to receiving adequate training. One patient recalled:

"I was pretty confident ... actually very confident ... like I said, it's not hard to use. And with all the-the training they showed me, and being able to do it in front of them before I was able to do it on my own analysis; ' one patient had previous experience of belimumab $S C$, prefilled syringe. $I V$ intravenous, $S C$ subcutaneous

helped ... with my comfort level. So once I did it at home, I was just ready to do it."

The questionnaire data showed that all patients were satisfied with using the autoinjector $(n=29$ [69\%] very satisfied; $n=11$ [26\%] satisfied; $n=2$ [5\%] somewhat satisfied). Qualitative interview responses $(n=21)$ showed that $17(81 \%)$ patients reported positive experiences using the autoinjector and five $(24 \%)$ patients reported having a negative experience $(n=2[10 \%]$ patients reported both positive and negative experiences). In the qualitative interviews, the most commonly reported advantages of the autoinjector were convenience $(n=15[71 \%])$, easy/quick administration $(n=12[57 \%])$, and self-administration $(n=5$ [24\%]). During the qualitative interviews, all patients considered the autoinjector to be convenient, citing shorter administration time compared with their previous treatment $(n=8[38 \%])$, ease of administration $(n=5$ [24\%]), ease of incorporation into their daily routine $(n=4[19 \%])$, and the ability to administer at home $(n=4[19 \%])$ as the main advantages. One patient stated,

"The convenience of just being able to go to the refrigerator, take it out, take a shower, get ready, 
Table 1 Patient demographics and clinical characteristics

\begin{tabular}{|c|c|c|}
\hline Characteristic & Questionnaire $(N=43)^{\mathrm{a}}$ & Qualitative interviews $(N=21)$ \\
\hline Age, years & $46.2 \pm 12.2$ & $47.6 \pm 12.6$ \\
\hline \multicolumn{3}{|l|}{ Sex } \\
\hline Male & $5(11.6)$ & $2(9.5)$ \\
\hline Female & $38(88.4)$ & $19(90.5)$ \\
\hline \multicolumn{3}{|l|}{ Race } \\
\hline White & $32(74.4)$ & $11(52.4)$ \\
\hline Black or African American & $9(20.9)$ & $9(42.9)$ \\
\hline Asian & $2(4.7)$ & $1(4.8)$ \\
\hline \multicolumn{3}{|l|}{ Employment status ${ }^{\mathrm{b}}$} \\
\hline Employed full time & $16(39)$ & $6(29)$ \\
\hline Employed part time & $5(12)$ & $2(10)$ \\
\hline Homemaker & $3(7)$ & $1(5)$ \\
\hline Unemployed & $1(2)$ & $1(5)$ \\
\hline Retired & $4(10)$ & $4(19)$ \\
\hline Disabled & $11(27)$ & $6(29)$ \\
\hline Other, self-employed & $1(2)$ & 0 \\
\hline \multicolumn{3}{|c|}{ Time since SLE diagnosis, years } \\
\hline$<1$ & $1(2)$ & 0 \\
\hline $2-5$ & $12(29)$ & $5(24)$ \\
\hline $5-10$ & $10(24)$ & $5(24)$ \\
\hline $10-15$ & $5(12)$ & $4(19)$ \\
\hline $15-20$ & $5(12)$ & $3(14)$ \\
\hline$>20$ & $6(15)$ & $4(19)$ \\
\hline \multicolumn{3}{|l|}{ Duration of belimumab use $\mathrm{c}^{\mathrm{c}}$} \\
\hline$<6$ months & $2(5)$ & $1(5)$ \\
\hline 6 months -1 year & $10(24)$ & $3(14)$ \\
\hline$>1$ year & $30(71)$ & $19(91)$ \\
\hline
\end{tabular}

Data are presented as mean \pm standard deviation or $n(\%)$

$S D$ standard deviation, $S L E$ system lupus erythematosus

${ }^{a}$ One patient was excluded from primary analyses due to completing the survey outside of the required time frame; data for this patient were only included in exploratory analyses

${ }^{\mathrm{b}} n=41$ for the questionnaire

${ }^{c} n=42$ for the questionnaire; first-year data blinded to patients who participated in study BEL112341

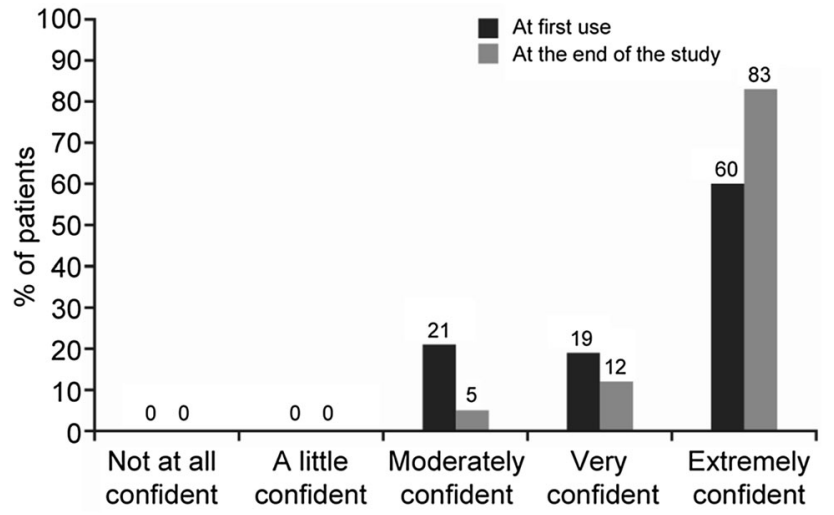

Fig. 2 Patient confidence in using the autoinjector alone, outside of the clinic (questionnaire results), $N=42$ come back out, give it to myself and be done with it. And not have to worry about missing work to do it, I can just do it in the morning as part of my routine and just do it and be done with it, that's the best part."

Furthermore, when specifically asked about ease of use, the majority of patients $(n=19[91 \%])$ reported that overall the autoinjector was easy to use. For example, one patient stated, "it was just super simple and so convenient for my daily life, that was my favorite things about it".

When asked about the disadvantages of the autoinjector, 8 of $21(38 \%)$ patients reported none and $13(62 \%)$ patients reported some, the most common of which was injection discomfort $(n=5 / 13$ [38\%]). These patients described pain, stinging, nausea, and a lack of control of injection 
speed. For example, “there wasn't much that I didn't like about it, except for that it stung. It was quick, it wasn't like intolerable, I could tolerate it, it didn't make me cry or it wasn't so much that it hurt, but it did sting".

In discussing their experience of nausea, one patient stated, "I had nausea ... for many days after, that was my only ... noticeable difference between the infusion and the injection".

Pain ratings for the autoinjector were low; on an 11-point numeric rating scale (from 0 [no pain] to 10 [extreme discomfort]), $35(83 \%)$ patients rated their discomfort as 0-3 (representing minimal pain), while three (7\%) patients indicated ratings of 4-6 (representing moderate pain), and four (10\%) patients indicated ratings of 7-8 (representing severe pain). Other cited disadvantages of the autoinjector included inconvenience (having to store the medication in a refrigerator and wait $30 \mathrm{~min}$ for it to reach room temperature before administration), not receiving enough medication, and it not being ergonomic ( $n=2 / 13$ for each [15\%]). Of the two patients who thought they did not receive enough medication, one stated the dose was not sufficient for their weight and another stated, "medicine needed to be a little stronger".

\subsection{Comparison of Routes of Administration of Belimumab}

A total of $32(76 \%)$ patients who switched from intravenous belimumab to autoinjector expressed in the questionnaire a preference for the autoinjector compared with intravenous administration. During subsequent telephone interviews, patients reported that convenience, time saved (including reduced travel time, quick administration compared with time taken for intravenous administration), cost, and reduced injection pain contributed to autoinjector preference.

Considering their prior experience with intravenous belimumab, one patient recalled,

"I mean, sometimes I'd feel that IV ... pull and stuff. And the site where the IV was is tender. And with that and with me being a hard person to get a vein on, sometimes I'm poked two or three times before the IV is even started."

One patient described adverse events with intravenous belimumab, "It-when I first went on the IV ... I started soon as I ... it started going in my system I would ... the itch-I would feel itchy like on my ... my face, my back, my stomach".

Patients who preferred intravenous administration perceived that, compared with the autoinjector, intravenous belimumab more adequately controlled their symptoms. One patient suggested,
"Well, right at this minute I prefer the IV, just because it makes me feel better. If they could get the autoinjector to where, you know, it makes you feel as good continuously like the IV did, then of course I would go with the autoinjector because of the convenience. But for-as of right this minute, I like the IV."

The questionnaire results indicate that overall satisfaction, satisfaction with frequency of administration, and satisfaction with time taken to receive belimumab were higher for the autoinjector than for intravenous administration (Table 2). The autoinjector was also reported to be more convenient than intravenous administration (Table 2). During the qualitative interviews, satisfaction was attributed to reduced time for autoinjector administration, less travel time and interference with work, autoinjector portability, and less/no pain associated with autoinjector use. All patients stated that the autoinjector was easier to use than receiving an intravenous infusion. Seven $(33 \%)$ patients indicated that it was easier to incorporate the autoinjector into their daily routine.

Patient-reported symptom changes varied when comparing the autoinjector and intravenous administration. Questionnaire results revealed that 17 (40\%) patients thought their symptoms were much or somewhat better, 14 (33\%) reported no change, and nine $(21 \%)$ reported they were much or somewhat worse with the autoinjector than with intravenous administration. During the qualitative interviews, 11 (52\%) patients reported no change in their symptom severity when using the autoinjector compared with intravenous administration; all five (24\%) of the patients who reported a decrease in symptom severity attributed this to greater symptom control because of consistency provided by weekly autoinjector administration compared with monthly intravenous infusions. For example, one patient commented,

"I feel like sometimes with the IV that the 4 weeks is a long time because like I said, before I go back with the, um, before the IV I have like my symptoms aren't very good - the lesions, the achiness, swelling.

But with this auto I didn't have that."

Four $(19 \%)$ patients reported experiencing less severe symptoms when using intravenous administration than when using the autoinjector.

Questionnaire responses revealed low levels of discomfort (on a scale of 0 [no discomfort]-10 [extreme discomfort]) for both autoinjector (mean [SD] 1.8 [2.2]) and intravenous administrations (mean [SD] 1.8 [1.9]). Of the three patients with experience of using the prefilled syringe, one reported that discomfort was greater with the autoinjector than with the prefilled syringe. During the 


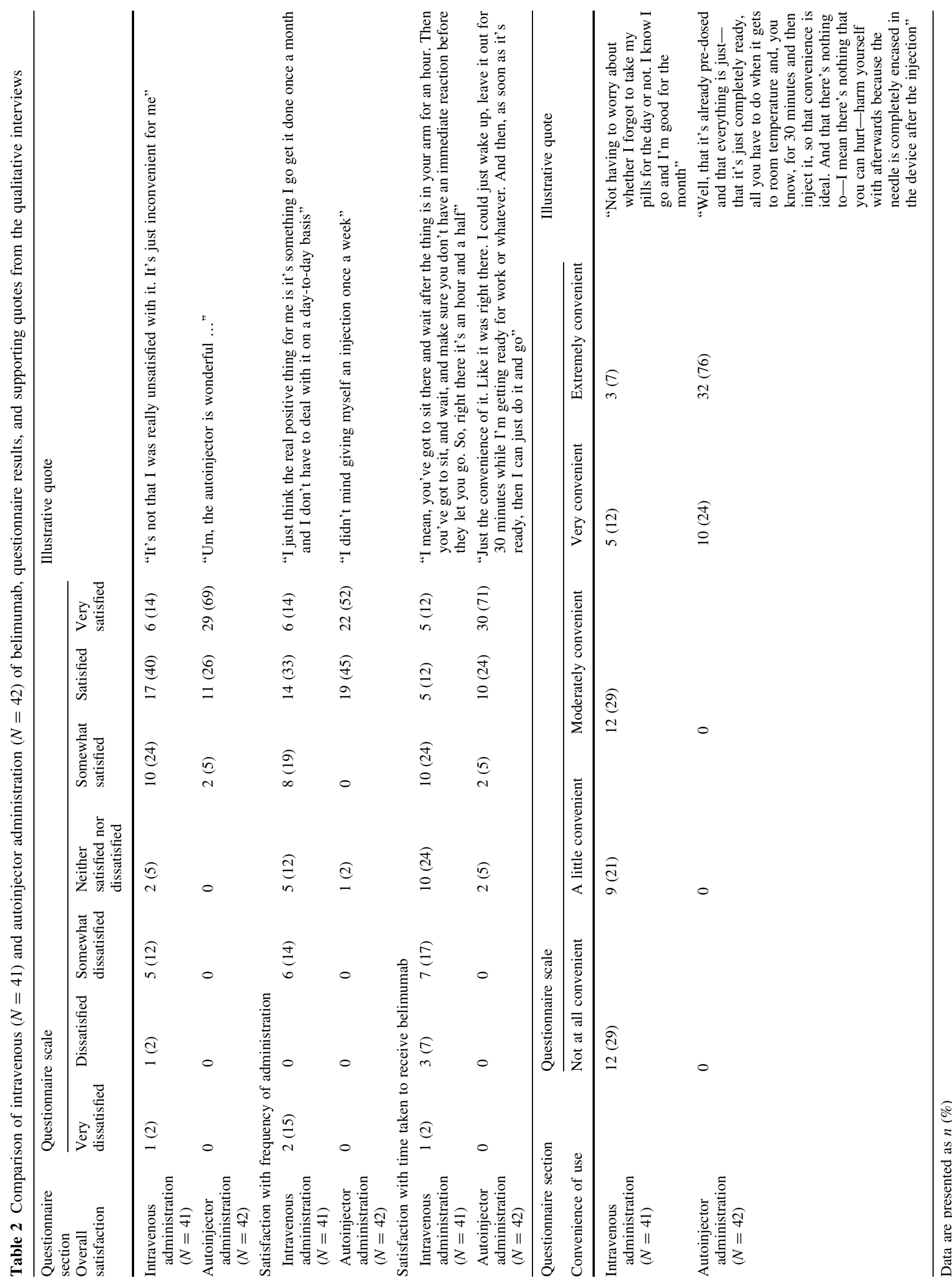


qualitative interviews, $17(81 \%)$ patients reported that the discomfort of using the autoinjector was the same as that experienced with intravenous infusions; two (10\%) reported greater discomfort and two (10\%) reported less discomfort than with intravenous administration. Overall, according to the questionnaire, pain ratings for both autoinjector and intravenous administration routes were low and comparable, with $35(83 \%)$ and $33(80 \%)$ patients rating their pain as minimal, respectively.

Questionnaire data indicated that $17(41 \%)$ patients thought autoinjector use improved their ability to carry out daily activities compared with intravenous administration; $18(44 \%)$ patients reported no change, and four $(10 \%)$ reported a decreased ability (data missing for $n=2$ [5\%] patients). For example, one patient stated:

"It's easier for me to do things, so I do more things ... I can do more varied things because I can walk for a longer period of time ... I can drive for longer periods of time, so it's made it easier to do more. So, it's made me happier which makes everyone else happier."

Among employed patients ( $n=29 / 42$ [69\%]), use of the autoinjector was reported to have a positive impact on their ability to work ( $n=17$ [59\%]) compared with intravenous administration; eight (28\%) patients reported no change and four (14\%) reported worsening of ability to work. Exploratory analyses investigated the relationship between ability to work and preference for route of administration; however, it is important to note that the subgroups for these analyses were small. Among employed patients who reported a preference for the autoinjector $(n=22 / 29$ [76\%]), $n=16$ / $22(73 \%)$ reported an improvement in their ability to work while using the autoinjector; the remaining patients in this subgroup reported no change. By comparison, only one (14\%) patient who reported a preference for intravenous belimumab reported an improvement in ability to work; over half of the patients who preferred intravenous belimumab compared with the autoinjector $(n=4 / 7$ [57\%]) reported that their ability to work had worsened with intravenous treatment.

\subsection{General Experience and Impact of Belimumab}

Patients were asked about their experience with belimumab since initiating treatment, regardless of the route of administration. A high level of satisfaction was reported for belimumab: questionnaire data revealed that $21(50 \%)$ patients were very satisfied, ten $(24 \%)$ were satisfied, and six $(14 \%)$ were somewhat satisfied with their treatment; one $(2 \%)$ was neither satisfied nor dissatisfied, none were dissatisfied, and two (5\%) were very dissatisfied. All patients stated they would have preferred to have initiated belimumab at an earlier stage of their disease, and all patients wanted to continue receiving belimumab. In the questionnaire, the majority of patients reported improvements in overall symptoms, flare frequency and severity, and fatigue with belimumab treatment in general (Table 3). Exploratory analyses showed that symptoms were more frequently reported as 'much improved' by patients who had received belimumab for $>1$ year $(n=18 / 30$ [60\%]) than by those who had received belimumab for $<1$ year $(n=3 / 13$ [23\%]). Similarly, results showed 'much improvement' in flare frequency $(n=16 / 30$ [53\%] vs. $2 / 13$ [15\%] patients), flare severity $(n=17 / 30$ [57\%] vs. $3 / 13$ [23\%]), and fatigue ( $n=10 / 30$ [33\%] vs. $1 / 13$ [8\%]) in patients receiving belimumab for $>1$ year compared with those receiving belimumab for $<1$ year. Similar analyses suggested that patients who reported improvements in flare severity or flare frequency were more satisfied with belimumab treatment.

During the qualitative interviews, the most commonly cited advantages of belimumab were improvements in symptoms/flares ( $n=10[48 \%])$, that it was more effective than other treatments $(n=5[24 \%])$, and that it improved HRQoL $(n=5[24 \%])$. The majority of patients $(n=17$ [81\%]) said there were no disadvantages to belimumab; however, three $(14 \%)$ thought it did not sufficiently alleviate symptoms and two (10\%) stated that cost was a disadvantage. For example, one patient said, "The only disadvantage that I have about it is, you know, it's-you know, with any other medication, the cost, that's the only thing, you know, is just the cost of it".

Questionnaire results also indicated improvements in HRQoL since initiation of belimumab, including ability to work (attendance/performance; $n=15[36 \%]$ ), personal relationships $(n=22[52 \%])$, leisure activities $(n=20$ [48\%]), and activities of daily living such as grooming, bathing and running errands $(n=29$ [69\%]). Eight (19\%) patients experienced no improvements in HRQoL following initiation of belimumab. Of the 30 patients receiving concomitant steroids, $20(67 \%)$ had reduced their steroid use since initiating belimumab treatment.

\section{Discussion}

This study provides evidence regarding general satisfaction and preferences of patients with SLE for belimumab treatment and autoinjector administration. The online/paper questionnaire and qualitative interview data demonstrate that patients with SLE who used the autoinjector to selfadminister belimumab in a trial designed to reflect realworld use found the autoinjector easy to use, viewed belimumab as effective for the treatment of SLE symptoms, and expressed a preference to continue belimumab 


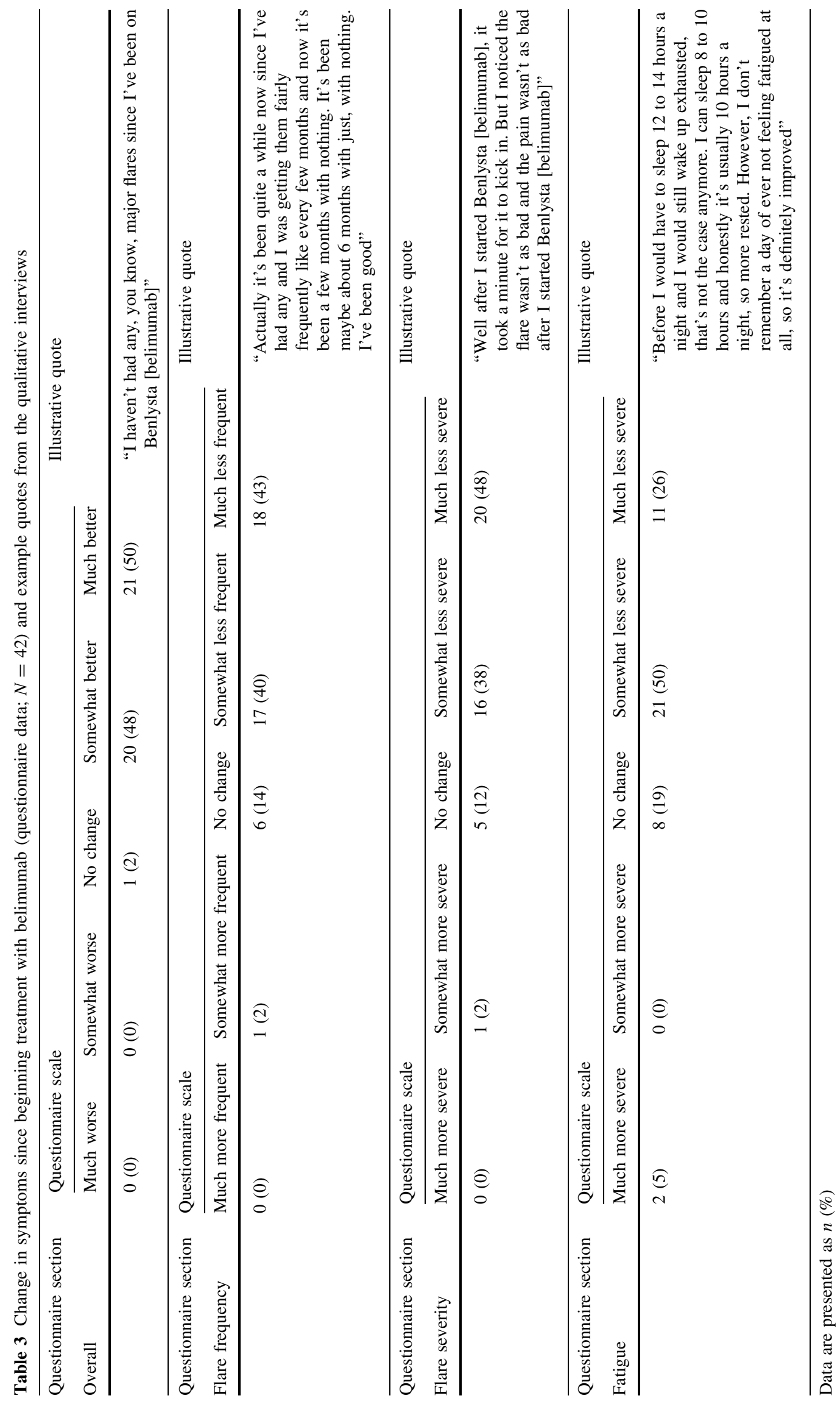


treatment if it was made available to them. Furthermore, $76 \%$ of patients expressed a preference for the autoinjector over intravenous administration for a variety of reasons, including convenience, time saved, cost, and reduced injection pain.

Patients were positive regarding their SLE symptom and HRQoL experiences when adding belimumab to SoC. Many patients reported that subcutaneous belimumab improved their ability to work and carry out daily activities. The positive experiences of patients reported here support the outcome of a physician and patient satisfaction survey of intravenous belimumab, in which good overall patient satisfaction with belimumab was demonstrated [2]. This study demonstrates that switching to autoinjector administration of belimumab also led to positive feedback in these areas. Furthermore, patients commonly reported satisfaction with the device, ease of use, convenience, and confidence with administration. Use of autoinjector devices has previously been shown as a strong predictor of drug adherence in patients with multiple sclerosis [11]. Improved patient satisfaction with subcutaneous belimumab administered via autoinjector may translate into improved adherence and consequential response to treatment.

Data from the present study regarding autoinjector ergonomics are consistent with those reported by healthy volunteers who found the device comfortable to hold [4]. Pain levels were low in the healthy volunteer study and in this study [4]. By comparison, injection discomfort was stated as a disadvantage in the present study by approximately one-quarter of patients. A small number of qualitative interview patients reported other disadvantages, including inconvenience, not receiving enough medication, and poor autoinjector ergonomics. Although one patient in this 8-week study thought the dose was insufficient due to their weight, data from the 52-week BLISS-SC trial demonstrated that the efficacy of subcutaneous belimumab was maintained across weight quartiles [6].

Patients expressed overall satisfaction with both the autoinjector and intravenous administration. Although patients did report some disadvantages, the majority indicated they generally preferred the autoinjector, with ease of administration, administration time, ease of incorporation into their daily routine, and ability to administer at home cited as key reasons. This is consistent with studies in patients with rheumatoid arthritis, which have also reported favorable patient evaluation of autoinjector devices [12-14].

Symptom improvements when using the autoinjector compared with intravenous administration were reported by $40 \%$ of patients in the questionnaire; telephone interviews revealed that symptom frequency was either consistent or improved in all patients who switched from intravenous administration to the autoinjector and that $24 \%$ of patients experienced a decrease in symptom severity. Treatment frequency was considered by some patients to be a further advantage; patients who reported a decrease in symptom severity with the belimumab autoinjector attributed this to improved symptom control consistency associated with weekly dosing.

Reported limitations of study NCT02124798 also affect this study [5]. Results from this study should also take into account limitations associated with self-selection bias, recall bias, unblinding, and concomitant medication use. Additionally, the majority of patients in the study population had been receiving belimumab for at least 1 year, so the experiences of patients with $<1$ year of treatment may not be adequately captured. The number of patients who switched from prefilled syringe to autoinjector device was low; therefore, no comparisons can be made between these modes of administration. However, a systematic review found that, in the majority of studies assessing patient preference for routes of treatment administration (including a study in patients with rheumatoid arthritis), patients preferred using an autoinjector device over a prefilled syringe, and the autoinjector device was associated with less injection site pain [3]. The overall sample size was adequate to achieve concept saturation (the point at which no new information is obtained from subsequent interviews); in qualitative studies with straightforward concepts, saturation is generally reached with a sample of 18-20 patients. However, the number of patients in the exploratory analyses subgroups was low, therefore, these results should be interpreted with caution.

The availability of the autoinjector for self-administration of subcutaneous belimumab provides an additional treatment option for patients with SLE, and results from this study clearly signal the potential benefits of belimumab and autoinjector use in SLE. The favorable opinion expressed by patients in this study reinforces the clinical improvements and HRQoL impacts of intravenous belimumab demonstrated in previous studies $[7,8,15]$, and suggests that patient outcomes may be improved by the use of the autoinjector.

\section{Conclusions}

Participants indicated a high level of satisfaction with the autoinjector. Results from this study suggest that patients would prefer to continue using belimumab treatment for SLE and that, compared with intravenous belimumab, the autoinjector has clear patient benefits and was the preferred administration route for three-quarters of patients. Further longitudinal studies will be conducted to confirm the findings of this cross-sectional study, to assess change in 
patient outcomes over time, and to evaluate the long-term use of the autoinjector device for the administration of belimumab to treat SLE.

Acknowledgements Medical writing assistance was provided by Katie White, $\mathrm{PhD}$, and Louisa Pettinger, $\mathrm{PhD}$, of Fishawack Indicia Ltd, and was funded by GlaxoSmithKline.

Author contributions ED-A and GH contributed to the conception and design of the study, acquisition of the data, and data analysis and interpretation. KP and PB contributed to the conception and design of the study, and data analysis and interpretation. JDV contributed to the conception and design of the study. SR contributed to the data analysis and interpretation. All authors were involved in drafting and revising the manuscript critically for important intellectual content and approved the final version for submission.

\section{Compliance with Ethical Standards}

Funding This study (HO14-15135, 201816) was conducted by Evidera, who received funding from GlaxoSmithKline. The parent study (200339, NCT02124798) was funded by GlaxoSmithKline.

Conflict of interest PB and SR are shareholders and employees of GlaxoSmithKline (GSK). GH is an employee of Evidera. ED-A was an employee of Evidera. at the time of the study. JDV is a shareholder of GSK, and both JDV and KP were employees of GSK at the time of the study.

Ethical approval All procedures performed in studies involving human participants were in accordance with the ethical standards of the institutional and/or national research committee and with the 1964 Declaration of Helsinki and its later amendments or comparable ethical standards.

Informed consent Informed consent was obtained from all individual participants included in the study.

Open Access This article is distributed under the terms of the Creative Commons Attribution-NonCommercial 4.0 International License (http://creativecommons.org/licenses/by-nc/4.0/), which permits any noncommercial use, distribution, and reproduction in any medium, provided you give appropriate credit to the original author(s) and the source, provide a link to the Creative Commons license, and indicate if changes were made.

\section{References}

1. GlaxoSmithKline. Benlysta Prescribing Information. Rockville: GlaxoSmithKline; 2017. hhttps://www.gsksource.com/pharma/ content/dam/GlaxoSmithKline/US/en/Prescribing_Information/ Benlysta/pdf/BENLYSTA-PI-MG-IFU-COMBINED.PDF. Accessed 22 Sept 2017.

2. Pascoe K, Lobosco S, Bell D, Hoskin B, Ramachandran S, Pobiner B, et al. Patient- and physician-reported satisfaction with systemic lupus erythematosus treatment in US clinical practice. Clin Ther. (Epub 10 August 2017).
3. Ridyard CH, Dawoud DMM, Tuersley LV, Hughes DA. A systematic review of patients' perspectives on the subcutaneous route of medication administration. Patient. 2016;9(4):281-92.

4. Struemper H, Murtaugh T, Gilbert J, Barton ME, Fire J, Groark J, et al. The relative bioavailability of a single dose of belimumab administered subcutaneously by prefilled syringe or autoinjector in healthy subjects. Clin Pharmacol Drug Dev. 2016;5(3):208-15.

5. Sheikh SZ, Hammer AE, Fox NL, Groark J, Struemper H, Roth $\mathrm{D}$, et al. Evaluation of a novel autoinjector for subcutaneous selfadministration of belimumab in systemic lupus erythematosus. Int J Pharmacol Ther. 2016;54(11):914-22.

6. Stohl W, Schwarting A, Okada M, Scheinberg M, Doria A, Hammer AE, et al. Efficacy and safety of subcutaneous belimumab in systemic lupus erythematosus: a fifty-two-week randomized, double-blind, placebo-controlled study. Arthritis Rheum. 2017;69(5):1016-27.

7. Furie R, Petri M, Zamani O, Cervera R, Wallace DJ, Tegzová D, et al. A phase III, randomized, placebo-controlled study of belimumab, a monoclonal antibody that inhibits B lymphocyte stimulator, in patients with systemic lupus erythematosus. Arthritis Rheum. 2011;63(12):3918-30.

8. Navarra SV, Guzmán RM, Gallacher AE, Hall S, Levy RA, Jimenez RE, et al. Efficacy and safety of belimumab in patients with active systemic lupus erythematosus: a randomised, placebo-controlled, phase 3 trial. Lancet. 2011;377(9767):721-31.

9. Mathias SD, Berry P, deVries J, Askanase A, Pascoe K, Colwell $\mathrm{H}$, et al. Development of two novel patient reported outcome (PRO) measures: the systemic lupus erythematosus (SLE) symptom severity diary (SSD) and SLE Impact Questionnaire (SIQ). Ann Rheum Dis. 2015;74(Suppl 2):560.

10. Mathias SD, Berry P, De Vries J, Askanase A, Pascoe K, Colwell $\mathrm{HH}$, et al. Development of the systemic lupus erythematosus Steroid Questionnaire (SSQ): a novel patient-reported outcome tool to assess the impact of oral steroid treatment. Health Qual Life Outcomes. 2017;15(1):43.

11. Pozzilli C, Schweikert B, Ecari U, Oentrich W. Supportive strategies to improve adherence to IFN beta- $1 \mathrm{~b}$ in multiple sclerosis-results of the BetaPlus observational cohort study. J Neurol Sci. 2011;307(1):120-6.

12. Kivitz A, Cohen S, Dowd JE, Edwards W, Thakker S, Wellborne FR, et al. Clinical assessment of pain, tolerability, and preference of an autoinjection pen versus a prefilled syringe for patient selfadministration of the fully human, monoclonal antibody adalimumab: The TOUCH trial. Clin Ther. 2006;28(10):1619-29.

13. Schulze-Koops H, Giacomelli R, Samborski W, Rednic S, Herold $\mathrm{M}$, Yao R, et al. Factors influencing the patient evaluation of injection experience with the SmartJect autoinjector in rheumatoid arthritis. Clin Exper Rheumatol. 2015;33(2):201-8.

14. Schwarzenbach F, Dao Trong M, Grange L, Laurent PE, Abry H, Cotten J, et al. Results of a human factors experiment of the usability and patient acceptance of a new autoinjector in patients with rheumatoid arthritis. Patient Prefer Adher. 2014;8:199-209.

15. Strand V, Levy RA, Cervera R, Petri MA, Birch H, Freimuth $\mathrm{WW}$, et al. Improvements in health-related quality of life with belimumab, a B-lymphocyte stimulator-specific inhibitor, in patients with autoantibody-positive systemic lupus erythematosus from the randomised controlled BLISS trials. Ann Rheum Dis. 2014;73(5):838-44. 